\title{
Hippocampal theta activity during delayed nonmatching-to-sample performance in rats
}

\author{
NOBUYA SATO and SHOGO SAKATA \\ Hiroshima University, Hiroshima, Japan
}

\begin{abstract}
Electroencephalograms (EEGs) were recorded from the rat hippocampal CA1, dentate gyrus, entorhinal cortex, and cingulate cortex during the performance of a delayed nonmatching-to-sample task. The recording of EEGs was divided into four task phases-control, sample, delay, and comparisonso that the relationship between the EEG and specific task events could be investigated. The amplitude of theta activity $(6-8 \mathrm{~Hz})$ in the hippocampal CAl increased during the comparison phase and increased differentially when rats made correct go response in the nonmatching trials. Between the hippocampal CAl and the entorhinal cortex, and the CAl and the cingulate cortex, EEG synchronization was higher in three task-related phases (sample, delay, and comparison) than in the control phase. These results suggest that hippocampal theta activity is involved in a mnemonic process of comparison between retained and current stimuli and that synchronized activity between the hippocampus and the related cortical structures was also involved in such a mnemonic process.
\end{abstract}

The hippocampus is probably involved in the processing of some types of memory, and these mnemonic processes can be divided into the hippocampal dependent and the hippocampal independent (see, e.g., Eichenbaum, Otto, \& Cohen, 1994; Olton, Becker, \& Handelmann, 1979; Squire, 1987, 1992; Sutherland \& Rudy, 1989). Hippocampal-dependent memory is necessary for solving delayed matching-to-sample (DMS) or delayed nonmatching-to-sample (DNMS) tasks involving a limited number of stimuli. Thus, rats with damage of the hippocampus could solve neither DMS nor DNMS tasks, whether spatial (Aggleton, Hunt, \& Rawlins, 1986) or nonspatial cues were used (Raffaele \& Olton, 1988).

The most characteristic electroencephalogram (EEG) of the hippocampus is the hippocampal theta activity, which is a high-amplitude, regular EEG pattern generated by dipoles in the CA1 subfield of the hippocampus or in the dentate gyrus (B. H. Bland \& Whishaw, 1976; Buzáki, 1986; Buzáki, Czopf, Kondákor, \& Kellényi, 1986; Buzáki, Leung, \& Vanderwolf, 1983; Lopes da Silva, 1992; Miller, 1991). There are several reports that suggest a relationship between hippocampal theta activity and mnemonic process. Winson (1978) abolished hippocampal theta activity by a lesion of the medial septal nucleus in the rat and found performance deficits in a spatial memory task. Givens and Olton $(1990,1994)$ blocked the hippocampal theta by locally administering cholinergic antagonists or GABAergic agonists to the medial septal nucleus. Ad-

The authors thank Iver $H$. Iversen for improving the English style of an early draft of the manuscript. Correspondence concerning this article should be addressed to S. Sakata, Department of Behavioral Sciences, Faculty of Integrated Arts and Sciences, Hiroshima University, 1-7-1 Kagamiyama, Higashi-Hiroshima, Hiroshima 739-8521, Japan (e-mail: ssakata@behavior.ias.hiroshima-u.ac.jp). ministration of these drugs impaired choice accuracy in a spatial delayed-alternation task and in the nonspatial, modified DNMS task. They also found a clear positive correlation between the power of hippocampal theta activity and choice accuracy in both the delayed-alternation task $(r=.78)$ and the DNMS task $(r=.61)$.

These studies, however, did not determine what kind of task demands relates to the hippocampal theta. The purpose of the present study was to examine this relationship between hippocampal theta activity and memory processes in the DNMS task. In particular, we investigated when the theta activity would be prominent during the sample, delay, and comparison phases of the task. Recordings were taken from the $\mathrm{CAl}$ subfield of the hippocampus, the dentate gyrus, the entorhinal cortex, the cingulate cortex, and the frontal cortex. Frontal EEG was used as an index of the arousal level. The reason we also recorded EEG from the entorhinal and cingulate cortices was that theta activity has been observed in the entorhinal cortex (Alonso \& García-Austt, 1987a, 1987b; Mitchell \& Ranck, 1980) and in the cingulate cortex (Feenstra \& Holsheimer, 1979; Leung \& Borst, 1987). In addition, both the entorhinal cortex (Montoya \& Sainsbury, 1985; Vanderwolf, Leung, \& Cooley, 1985) and the cingulate cortex (Destrade \& Ott, 1982; Vanderwolf et al., 1985) must be intact for the generation of hippocampal theta (especially higher frequencies). Thus, we investigated the interaction reflected in the theta activity of the entorhinal cortex, the cingulate cortex, and the hippocampal formation during DNMS performance.

\section{METHOD}

\author{
Subjects \\ Eleven male albino rats, $3-5$ months old, were used. The rats \\ were housed individually, with a 12:12-h light:dark cycle. Lights
}




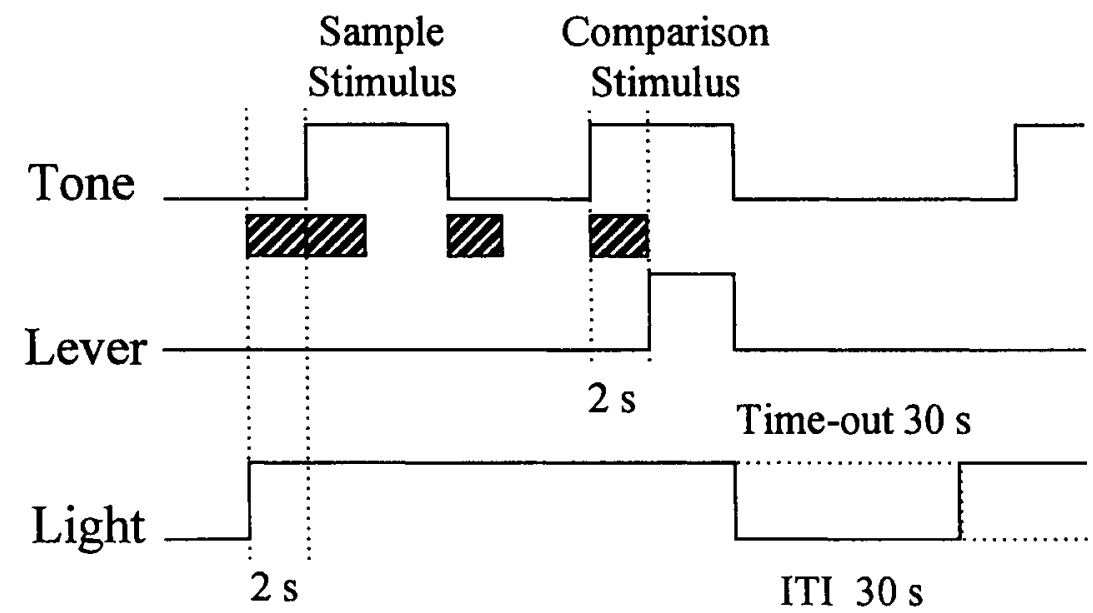

\title{
EEG analysis period
}

\begin{abstract}
Figure 1. Temporal sequence of the present delayed nonmatching-to-sample task. This scheme shows one trial sequence in the task. Each line indicates tone presentation, insertion of the lever, and an illumination of the houselight. If animals pressed the lever on the match trial, the lever was retracted but the houselight remained lit for $30 \mathrm{sec}$ as a time-out period (broken line in the houselight sequence). After this, the intertrial interval began. Recordings of electroencephalograms were analyzed at four phases, shown by oblique stripes for the control, sample, delay, and comparison phases, respectively.
\end{abstract}

were on from 9:00 a.m. to 9:00 p.m. All the behavioral training and recording sessions were conducted during the light phase of the cycle. All the rats were maintained at $90 \%$ of their ad-lib weights. Water was available continuously.

\section{Apparatus}

Behavioral training and recording sessions took place in an operant chamber, $25 \times 20 \times 38 \mathrm{~cm}$. A cup for delivery of food pellets was located in the center of the front wall at the level of the floor. A retractable response lever (Coulbourn Instruments, Allentown, PA) was positioned on the right side of the front wall, $3 \mathrm{~cm}$ from the cup and $4.5 \mathrm{~cm}$ above the floor. A loudspeaker was mounted on the front wall, $10 \mathrm{~cm}$ above the cup. An $8-\mathrm{V} \mathrm{dc}$ bulb was mounted on the ceiling as a houselight. A food dispenser delivered 45-mg food pellets to the cup. The rats' behavior was monitored by means of a closed-circuit TV system. The chamber was enclosed in a soundproof, electrical shielding box (Japan Shield Enclosure, Inc., Osaka, Japan). All events were controlled and behavioral data recorded by a personal computer (NEC, Tokyo, Japan).

\section{Procedure}

The rats were trained to perform a successive DNMS task that used two pure tones $(2 \mathrm{kHz}$ or $8 \mathrm{kHz}$, both at $80 \mathrm{~dB}$ ). Figure 1 shows the temporal sequence of the task. At the start of each trial, the houselight was illuminated. After $2 \mathrm{sec}$, one of the two tones was presented for $5 \mathrm{sec}$ as a sample stimulus, followed by a 1- or a 5 -sec delay period. At the end of the delay period, one of the same two tones was presented for $5 \mathrm{sec}$ as the comparison stimulus. The lever was inserted into the chamber $2 \mathrm{sec}$ after onset of the comparison stimulus. Pressing the lever caused elimination of the comparison stimulus and retraction of the lever (a go response). If the rat did not press the lever within $3 \mathrm{sec}$ from insertion of the lever, the comparison stimulus was eliminated, and the lever was retracted (a no-go response).

The go response on nonmatching trials, in which the comparison stimulus was different from the sample stimulus, was reinforced. The go response on matching trials, in which the comparison stim- ulus was the same as the sample stimulus, was not reinforced and caused a $30-\mathrm{sec}$ time-out period. The no-go response during both trial types was not reinforced and did not cause a time-out period. Each trial was followed by a 30 -sec intertrial interval, during which the houselight was turned off. Each daily training and recording session consisted of 90 trials, with 30 nonmatch trials and 60 match trials. After box habituation and shaping to press the lever, each rat was trained in the above task with a 1-sec delay period for 30 sessions, and then with a 5 -sec delay period for 20 sessions. After this training, electrodes for EEG recording were implanted in each rat. The 5-day recording sessions were carried out at least 5 days after the surgery.

\section{Surgery}

After anesthesia with thiamylal sodium ( $40 \mathrm{mg} / \mathrm{kg}$, i.p.), each rat was placed in the stereotaxic apparatus (Narishige Scientific Inst. Lab., Tokyo, Japan). The electrodes were implanted stereotaxically in five regions. The stereotaxic coordinates for each region were as follows: Hippocampal CA1, $3.5 \mathrm{~mm}$ posterior from bregma, $2.0 \mathrm{~mm}$ lateral from the midline, $2.4 \mathrm{~mm}$ beneath the skull surface; dentate gyrus, $5.0 \mathrm{~mm}$ posterior from bregma, $3.0 \mathrm{~mm}$ lateral from the midline, $3.0 \mathrm{~mm}$ beneath the skull surface; entorhinal cortex, $8.8 \mathrm{~mm}$ posterior from bregma, $5.3 \mathrm{~mm}$ lateral from the midline, $5.0 \mathrm{~mm}$ beneath the skull surface; cingulate cortex, $5.5 \mathrm{~mm}$ posterior from bregma, $0.5 \mathrm{~mm}$ lateral from the midline, $1.5 \mathrm{~mm}$ beneath the skull surface; and frontal cortex, $4.0 \mathrm{~mm}$ anterior from bregma, $1.5 \mathrm{~mm}$ lateral from the midline, and near the dura beneath the skull surface.

The electrodes for EEG recording were stainless steel wire, 200$\mu \mathrm{m}$ diameter, insulated with enamel (MT Inst., Tokyo, Japan). A silver ball electrode insulated with enamel (Narishige Scientific Inst. Lab., Tokyo, Japan) was placed into the nasal skull as a reference. A lead wire that had a 5-mm-diameter disk of solder on the tip was inserted under the neck skin as a body ground. There has been no evidence that this method for body ground causes an allergic reaction, an infection, or abnormal rats' behavior. Five stainless steel screws ( $1-\mathrm{mm}$ diameter) were placed into the skull. The electrodes were connected to a nine-pin connector (Amphenol, Wall- 


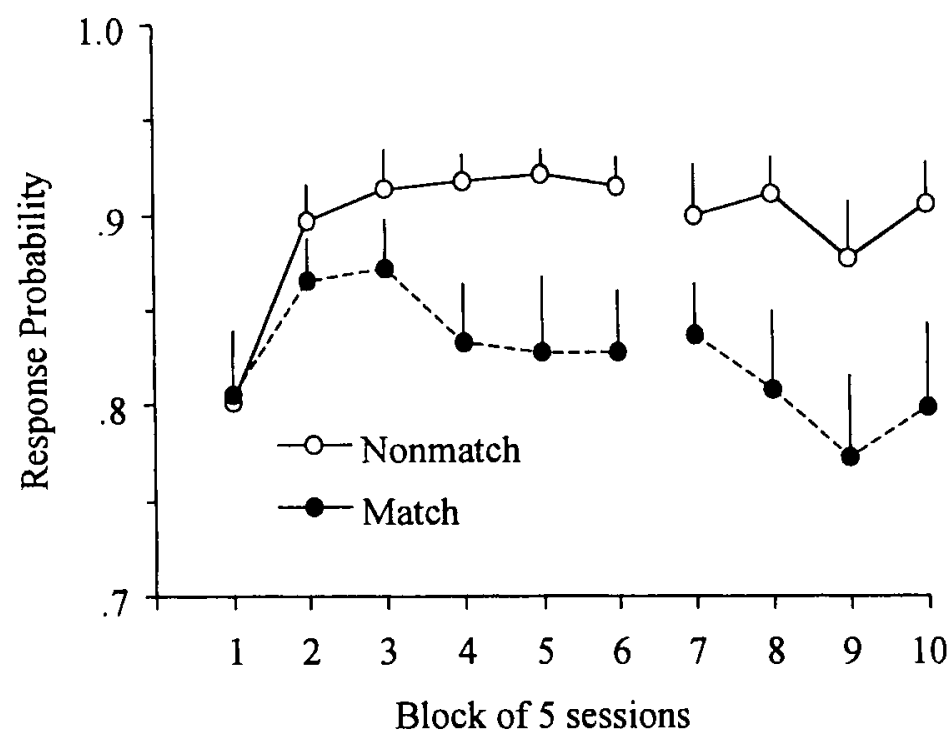

Figure 2. Mean response probabilities of blocks of five sessions in the nonmatch and match trials. The closed and open circles indicate the response probabilities in the nonmatch and match trials, respectively. After the seventh block, the delay period was changed to 5 sec from 1 sec. Vertical bars indicate the standard errors of the mean.

ingford, CT) and fixed with dental cement to the screws and the skull.

\section{Electroencephalogram Recording and Analysis}

The signals derived from the electrodes passed through an eightchannel preamplifier and were fed into a polygraph (NEC-Sanei, Tokyo, Japan) and stored on magnetic tape for off-line analysis (TEAC, Tokyo, Japan). The present study intended to investigate the relationship between the hippocampal theta activity and the DNMS task events. For this purpose, we analyzed EEGs during four task phases: control, sample, delay, and comparison (Figure 1). The control phase was the period from houselight illumination to the onset of the sample stimulus. During this phase, it was expected that the rats were not processing any mnemonic or task-related information. The sample phase was the first $2,048 \mathrm{msec}$ of the sample stimulus. The delay phase was the first $2,048 \mathrm{msec}$ of the delay period. The comparison phase was the first $2,048 \mathrm{msec}$ of the comparison stimulus. Because the lever was retracted, the rats could not press it during this phase. The EEGs of the four phases were digitized at a sampling rate of $250 \mathrm{~Hz}$ after analog bandpass filtering of between 0.5 and $30 \mathrm{~Hz}$. Phases with artifacts, according to visual inspection, were rejected. Using the fast Fourier transform, the spectrum of each phase for power and coherence was computed with a frequency resolution of $0.5 \mathrm{~Hz}$. To obtain the coherence function, the cross-spectrum had to be computed. We then calculated a peak of the cross-spectrum within the hippocampal theta frequency band $(4-12 \mathrm{~Hz})$ and analyzed statistically the coherence values at the frequencies of this cross-spectral peaks. For the statistical analyses, the coherence data were transformed to Fisher's $z$ score, to ensure their normal distribution.

\section{Histology}

At the end of the experiment, all the rats were deeply anesthetized with an overdose of thiamylal sodium $(200 \mathrm{mg} / \mathrm{kg}$, i.p. $)$ and perfused with a $10 \%$ buffered formalin solution. The brains were removed from the skulls and stored in a $10 \%$ formalin solution for 1 week. Then they were frozen and sectioned at $70-\mu \mathrm{m}$ intervals and stained with cresyl violet. The location of the electrode tips in the brain were identified in accordance with a stereotaxic atlas of rats (Paxinos \& Watson, 1986).

\section{RESULTS}

\section{Behavior}

At the beginning of the DNMS training, we could not distinguish the rats' behavior between the nonmatch and the match trials. Gradually, as the training session proceeded, the rats made more go responses in the nonmatch trials than in the match trials (Figure 2). As a result of a two-way (trial type $\times$ block of five sessions) repeated measures analysis of variance (ANOVA), a significant trial type effect $[F(1,10)=11.34, p<.01]$ and a trial $\times$ block interaction $[F(5,50)=4.73, p<.01]$ were obtained. Subsequent analyses of simple main effects revealed that there were significant differences in response probabilities between the nonmatch and the match trials after the fourth block of five sessions $(p s<.05)$. We therefore considered that the rats discriminated between the nonmatch and the match stimuli. In addition, we found no consistent difference in overt behavior during the EEG recording periods among the four task phases and between the nonmatch and the match trials.

\section{Electroencephalogram-Recorded Sites}

Figure 3 shows the anatomical locations of the tips of the electrodes for the EEG recording, except for the frontal 

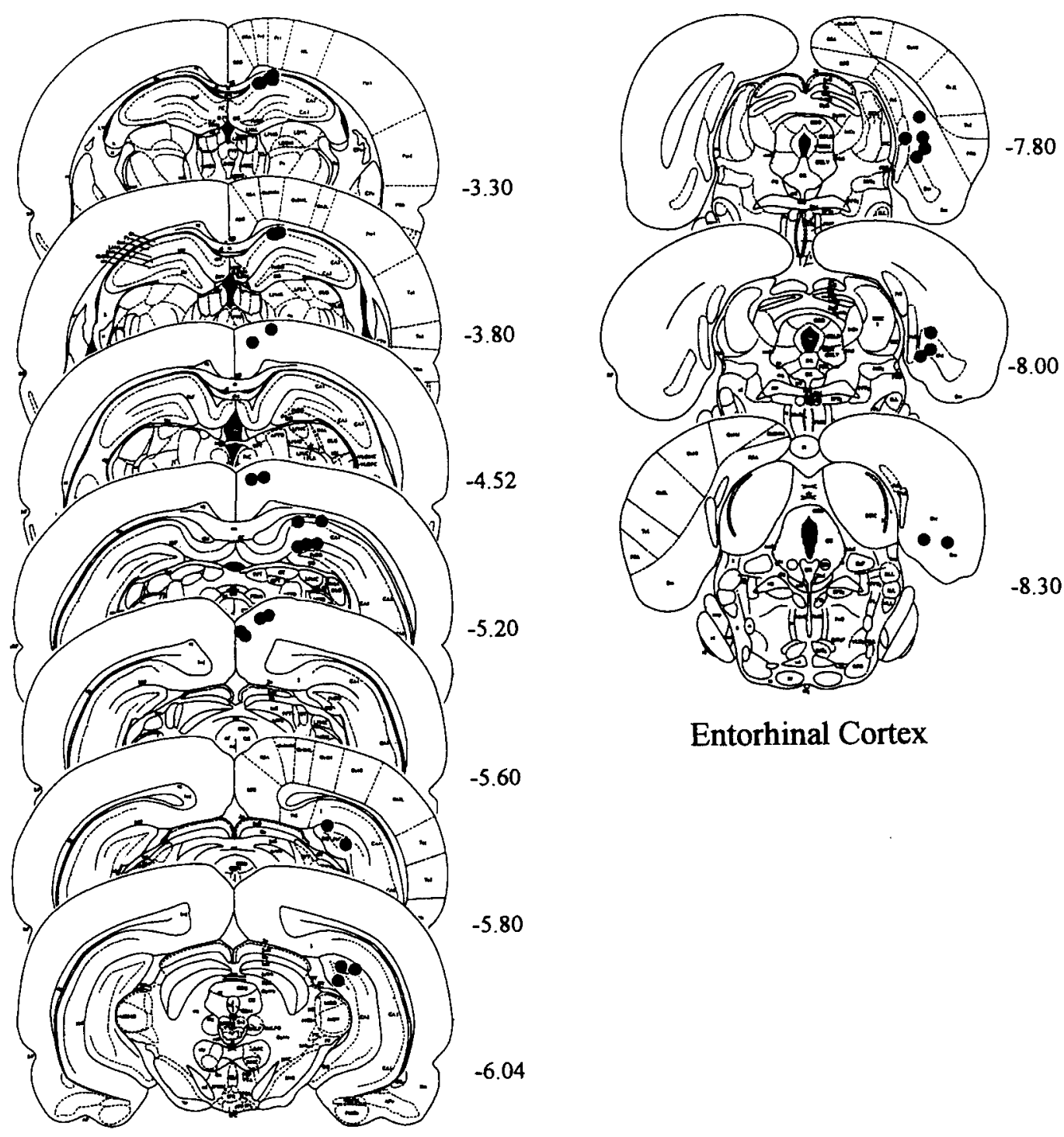

Entorhinal Cortex

\section{Hippocampal CAl \\ Dentate Gyrus \\ Cingulate Cortex}

Figure 3. Anatomical locations of the tips of electrodes, plotted on the transverse section outlines. The numbers beside each section indicate the distance from bregma in millimeters. From The Rat Brain in Stereotaxic Coordinates (2nd ed.; Figures 31, 33, 36, 38, 40, 41, 42, 49, 50, and 51) by G. Paxinos and C. Watson, 1986, San Diego, CA: Academic Press. Copyright 1986 by Academic Press. Reprinted with permission.

cortex sites. The following analyses were carried out on recordings derived from 12 hippocampal CA1, 8 dentate gyrus, 10 cingulate cortex, 10 entorhinal cortex, and 11 frontal cortex sites.

\section{Hippocampal Theta Activity}

Figure 4 shows the power spectra in the hippocampal CAl (A) and the dentate gyrus (B) during the four task phases. In both regions, the peaks of the power that cor- responded to hippocampal theta activity during all the recorded phases could be observed. In the hippocampal $\mathrm{CAl}$, theta activity gradually increased as the trial sequence went on and was most prominent in the comparison phase (Figure 5). A one-way repeated measures ANOVA for the $6-8 \mathrm{~Hz}$ band power revealed a significant difference between phases $[F(3,33)=10.19, p<.01]$. Subsequent Newman-Keuls tests indicated that the powers during the delay and the comparison phases were signifi- 

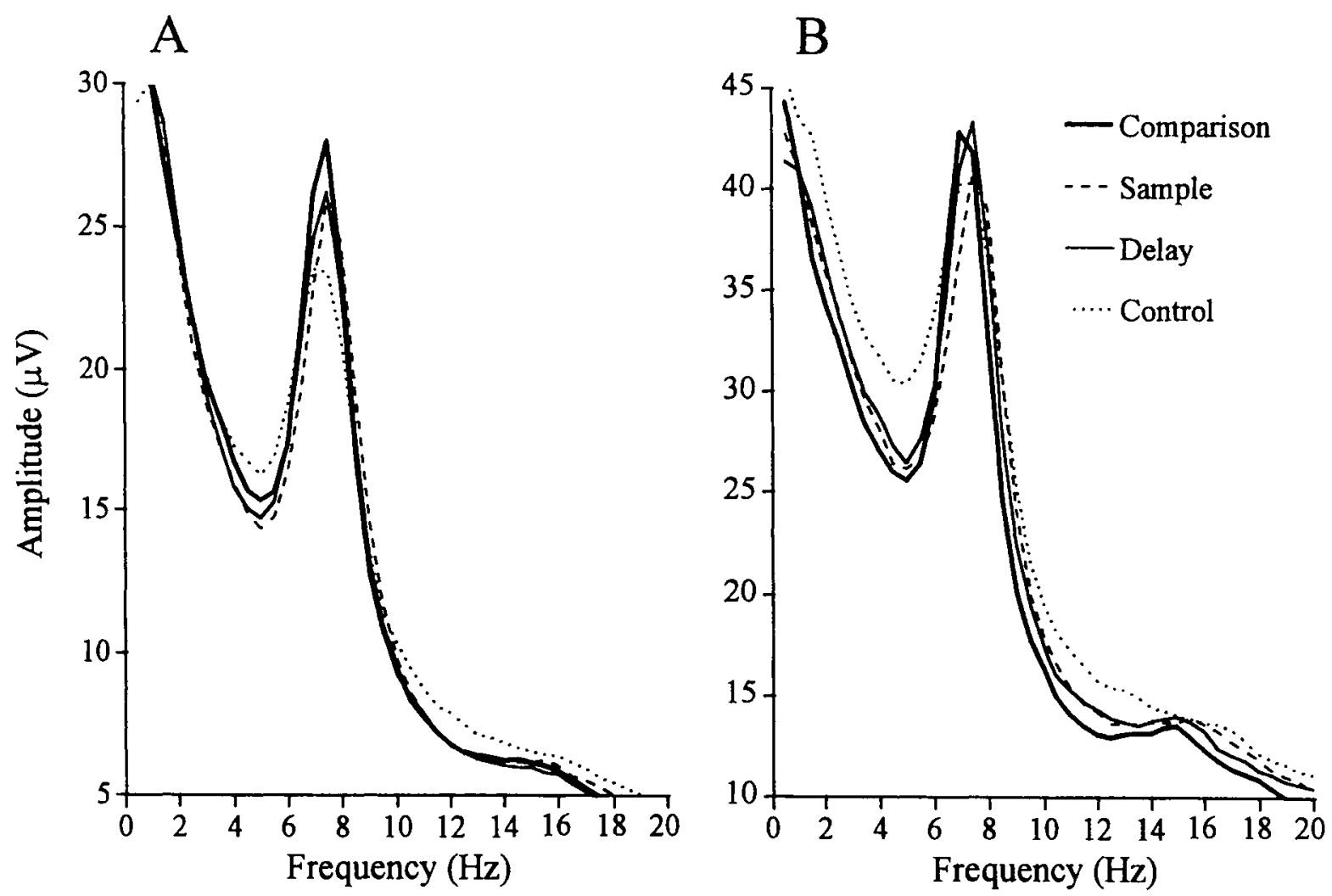

Figure 4. Mean power spectra in the hippocampal CA1 (A) and the dentate gyrus (B) during the four task phases: control (dotted line), sample (broken line), delay (thin line), and comparison (thick line).

cantly larger than those during the control and the sample phases $(p s<.05)$. A similar ANOVA for the dentate gyrus revealed no significant differences between phases.

The subjects were divided into two groups, according to their performance. Then the difference of theta activity of the hippocampal CA1 in the comparison phase from that in the control phase was compared between the two groups. As a result of this, it was found that theta activities of the higher performance group tended to increase more in the comparison phase than those of the lower performance group $[t(10)=2.05, p<.07]$. This suggests that the increase of theta amplitude in the hippocampal CA1 was performance dependent.

During the comparison phase, it was expected that the rats would compare the comparison stimulus with the mnemonic representation of the sample stimulus. It is likely that an increase of CAl theta activity is involved in such a comparison process. To examine this possibility, we further analyzed the CAl theta activity during the comparison phase. Initially, we compared the $6-8 \mathrm{~Hz}$ power during the comparison phase in the nonmatch trials with that in the match trials (Figure 6A). A paired twotailed $t$ test showed that the $6-8 \mathrm{~Hz}$ power of the hippocampal CA1 was larger in the nonmatch trials than in the match trials $[t(11)=2.88, p<.05]$. Vanderwolf (1969), however, reported that hippocampal theta activity was involved in voluntary movements. This nonmatch/ match difference could be attributed to the difference in the leverpressing response, because the proportion of leverpressing in the nonmatch trials was higher than that in the match trials. Next, we compared the EEG power during the comparison phase between the nonmatch go (nonmatch trials in which the rats pressed the lever), the match go (match trials in which the rats pressed the lever), and the match no-go trials (match trials in which the rats did not press the lever; Figure 6B). The power in the nonmatch no-go trials (nonmatch trials in which the rats did not press the lever) was not analyzed, because of an insufficient number of trials. A one-way ANOVA with repeated measures revealed a significant difference in the trial type $[F(2,22)=4.05, p<.05]$. Subsequent NewmanKeuls tests indicated that the $6-8 \mathrm{~Hz}$ power in the nonmatch go trials was larger than those in the match go trials and in the match no-go trials $(p$ s $<.05)$. Similar analyses were also conducted for the dentate theta activ. ity, but no differences among the trial types were found.

\section{The Interaction Between the Hippocampus and the Related Cortical Structures}

To estimate the mutual relationships among signals derived from the hippocampal formation and the related cortical structures, we computed the squared coherence 


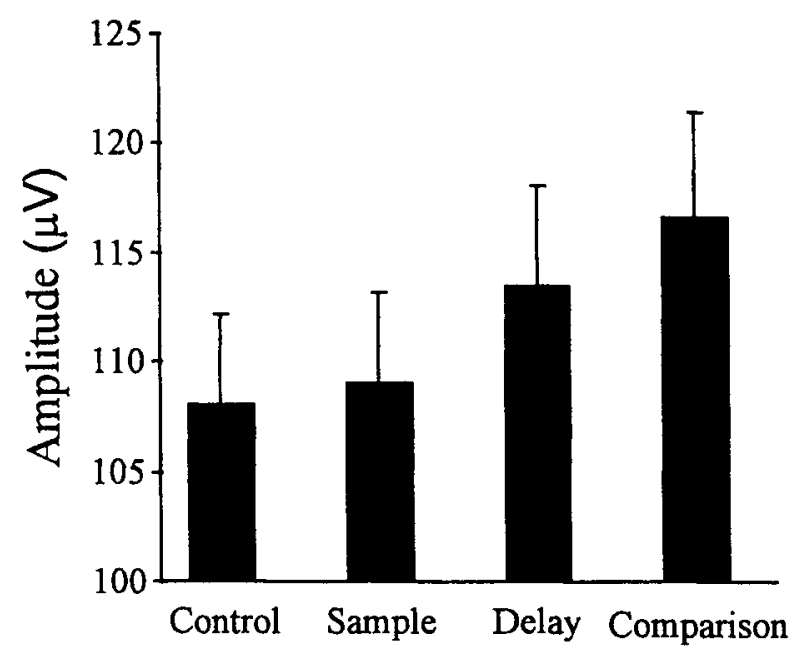

Figure 5. Mean amplitudes of theta activity measured by $6-8 \mathrm{~Hz}$ band power in the hippocampal CA1 during the four task phases. Vertical error bars indicate the standard errors of the mean.

values at the frequencies of the cross-spectral peaks within the theta frequency band $(4-12 \mathrm{~Hz})$. Inasmuch as power spectral peaks within the theta frequency range were also observed in the entorhinal and cingulate cortices (data not shown), a validity and significance of the present coherence was manifested. Figure 7 shows the coherence values during the control, sample, delay, and comparison phases. In each pair, the coherence value during the sample, delay, or comparison phase was larger than that during the control phase. Between the hippocampal CA1 and the entorhinal cortex, a one-way ANOVA with repeated values revealed a significant difference in the coherence values $[F(3,30)=5.50, p<.01]$. Subsequent NewmanKeuls tests indicated that the coherence values during the sample, delay, and comparison phases were larger than that during the control phase $(p \mathrm{~s}<.05)$. Between the hippocampal CA1 and the cingulate cortex, a one-way ANOVA also revealed a significant difference in the coherence values $[F(3,30)=4.42, p<.05]$. Subsequent Newman-Keuls tests indicated that the coherence values during the sample, delay, and comparison phases were larger than that during the control phase $(p s<.05)$. Between the dentate gyrus and the cingulate cortex, a oneway ANOVA also revealed a significant difference in the coherence values $[F(3,18)=6.93, p<.01]$. Subsequent Newman-Keuls tests indicated the coherence values during the sample, delay, and comparison phases were larger than that during the control phase $(p s<.05)$. However, for the dentate gyrus and the entorhinal cortex, a similar ANOVA revealed no significant difference between the coherence values.

\section{Beta Band Electroencephalogram Activity in the Frontal Cortex}

To investigate the arousal effects, we calculated the beta band $(13-20 \mathrm{~Hz})$ EEG activities of the frontal cortex and compared them among the four task phases. A oneway repeated measures ANOVA revealed a significant difference between the task phases $[F(3,30)=7.02, p<$ $.01]$. Subsequent Newman-Keuls tests indicated that beta power during the control phase was significantly larger than that during the sample, delay, and comparison phases $(p s<.05)$. This indicates that the rat was in an arousal state during the control phase, as compared with the other task-related phases.

\section{DISCUSSION}

The results of the present experiment demonstrate that the hippocampal theta activity has a significant correla-
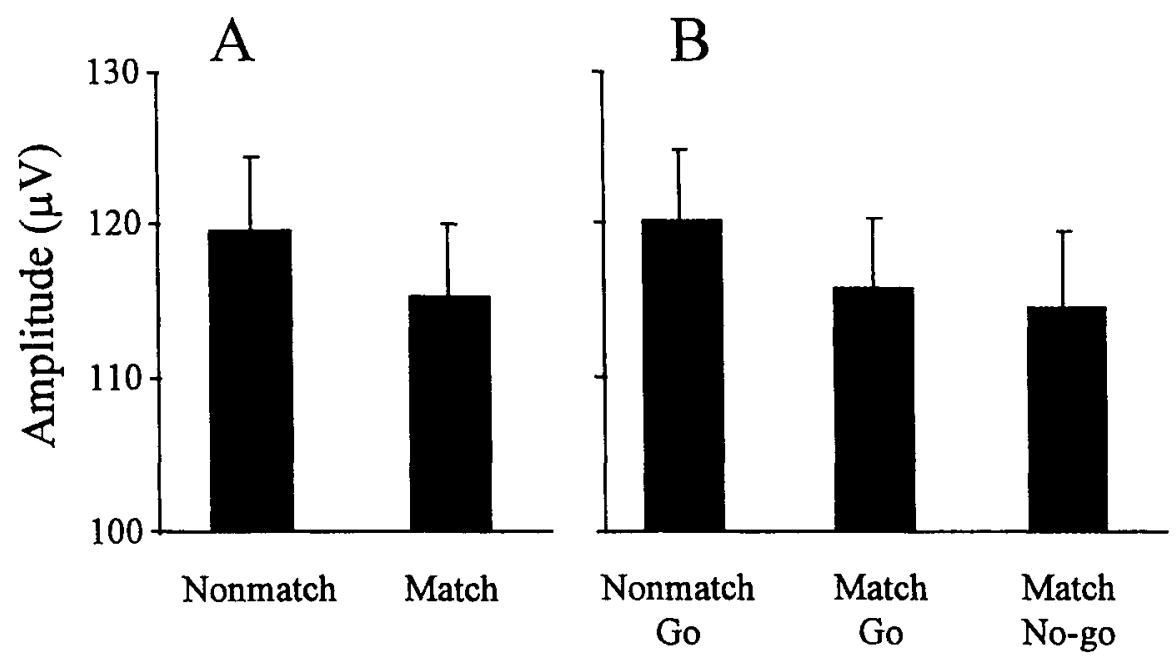

Figure 6. Mean amplitudes of theta activity in the hippocampal CA1 during the comparison phase. Panel A: Comparison was made between the theta activity in the nonmatch and match trials. Panel B: Theta activities were compared between the nonmatch go, the match go, and the match no-go trials. Vertical error bars indicate the standard errors of the mean. 

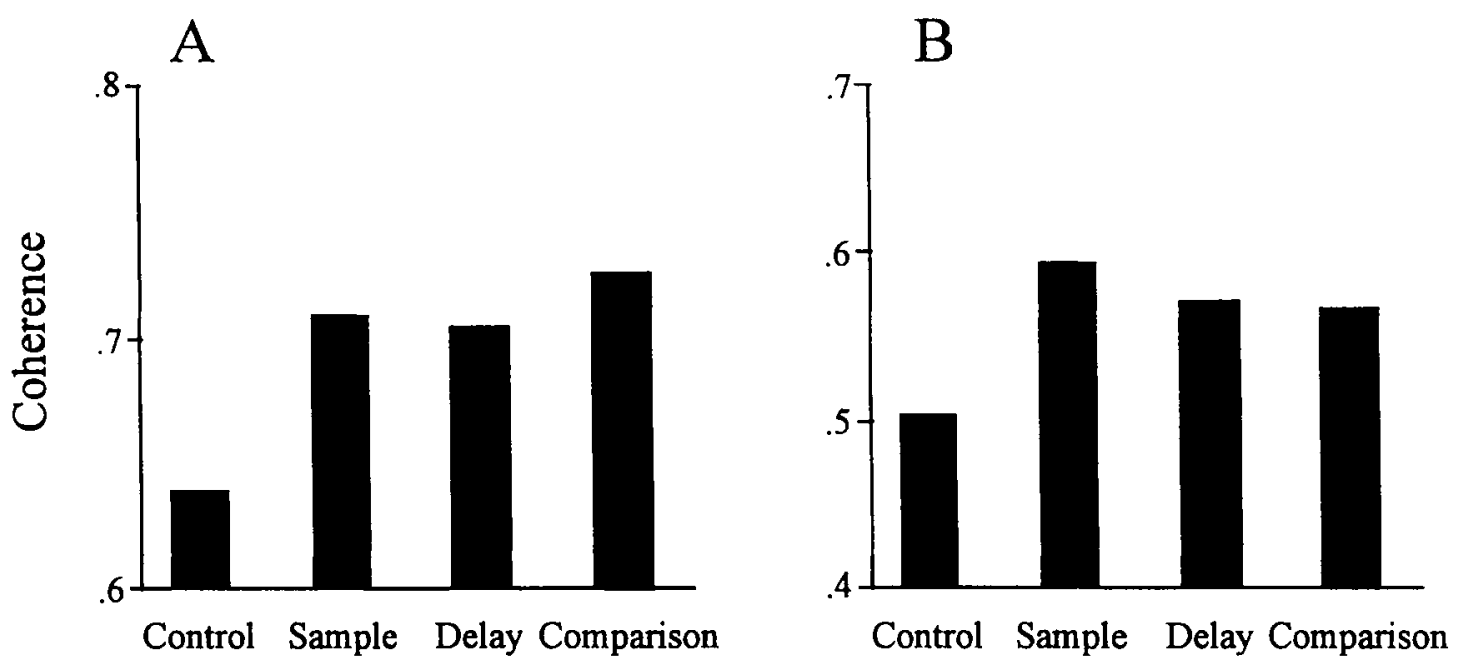

Figure 7. Coherence values between the hippocampal CA1 and the entorhinal cortex $(A)$ and between the hippocampal CA1 and the cingulate cortex (B) during the four task phases.

tion with the DNMS task events. This correlation is consistent with previous studies that reported that blocking of the hippocampal theta resulted in impairment of choice accuracy in spatial (Givens \& Olton, 1990; Winson, 1978) and nonspatial (Givens \& Olton, 1994) memory tasks. These previous studies, together with the present results, suggest that hippocampal theta activity reflects some aspects of the mnemonic functions of the hippocampus.

The present results suggest that hippocampal theta activity is involved in a mnemonic process through which stored information is compared with current events and behavioral response is then controlled on the basis of the comparison. The amplitude of theta activity in the hippocampal CAl increased during the comparison phase, as compared with the control phase, and increased when the rats made a correct go response in the nonmatch trials, as compared with when the rats made an incorrect go response in the match trials. These data were consistent with the research effort conducted to examine a mnemonic correlation with hippocampal neuronal activity. In a modified DNMS task, hippocampal cells are also differentially active when animals make a correct go response as a result of a comparison of stored stimuli with current ones (Otto \& Eichenbaum, 1992b; Sakurai, 1990b).

However, we cannot completely reject the possibility that the increased theta amplitude in the present study is involved in something other than the mnemonic process. It has been well known that hippocampal theta activity is involved in voluntary movements (Vanderwolf, 1969) and that leverpressing is accompanied by theta activity (Frederickson \& Whishaw, 1977; Young, 1976). In the present study, it was found that CAl theta activity in the comparison phase increased more in the nonmatch trials than in the match trials. These differences might have been involved in the preparation of a leverpressing response, although the rats actually could not press the lever during the EEG analysis period in the comparison phase. Thus, we made a comparison of the theta activity among the nonmatch go, the match go, and the match no-go trials and found that theta power in the nonmatch go trial was larger than that in the match go and match no-go trials. The rats made a go response in both the nonmatch go and the match go trials, but the context of the comparison stimulus was different between the two trials. Nevertheless, the fact that a go response is made on both types of trials does not mean that it is made with the same force that might influence the theta activity. Only, from the present study, it is impossible to dissociate a mnemonic process that consists of a comparison between stored and current information and a control of the behavioral response from a preparation process of the behavioral response itself. Further studies are needed to solve this problem.

A difference of arousal level may also influence the present results. The beta power of the frontal cortex was higher in the control phase than in the sample, delay, and comparison phases. An illumination of the houselight perhaps caused this result (Troncoso, Rodríguez, \& Feria, 1995). Because the intertrial interval of the task was relatively long ( $30 \mathrm{sec}$, and a $30-\mathrm{sec}$ time-out period was added next to an incorrect go trial), it was likely that the light illumination transiently raised the arousal level of the rat. However, this result at least indicates that the increase of CAl theta amplitude in the comparison phase was not due to the arousal state of the rat.

The theta activity in the dentate gyrus was different from that in the hippocampal CA1. Although high-amplitude dentate theta activity was observed, there were no differences between the behavioral phases in the task, between the nonmatch and the match trials, or among the nonmatch go, the match go, and the match no-go trials. These results are consistent with studies that examined the effects of septal or fornix-fimbria lesions on CA1 and dentate theta 
activities (M'Harzi \& Monmaur, 1985; Monmaur, 1982; Sainsbury \& Bland, 1981). These studies showed that fornix or lateral septal lesions disrupted mainly the CA1 theta, but not the dentate theta, and that fimbria or medial septal lesions disrupted inversely the dentate theta but did not disrupt the CA1 theta. Green and Rawlins (1979) showed precedent appearance of the CAl theta to the dentate theta in response to brain stimulation. These studies and the present results suggest that theta generators in the hippocampal $\mathrm{CAl}$ and the dentate gyrus are independent. In addition, the present results are also consistent with the fact that neurons with activities that reflect a comparison between current and stored events are much rarer in the dentate gyrus than in the hippocampal CAl (Sakurai, 1990b). These data probably reflect a functional difference between the hippocampal CAl and the dentate gyrus.

We suppose that interactions between the hippocampus and the entorhinal cortex and the hippocampus and the cingulate cortex are important for a mnemonic function of the hippocampus. To generate the hippocampal theta activity, intact medial septum/diagonal band is needed (S. K. Bland \& B. H. Bland, 1986; Gray, 1971; Winson, 1978). In addition, intact entorhinal (Montoya \& Sainsbury, 1985) and cingulate (Destrade \& Ott, 1982; Vanderwolf et al., 1985) cortices and connections between the entorhinal cortex and the neocortex (Vanderwolf et al., 1985) are needed for normal hippocampal theta activity. There were robust connections between the hippocampus and the entorhinal cortex, the hippocampus and the cingulate cortex, and the hippocampus and the neocortex in rats (Ferino, Thierry, \& Glowinski, 1987; Swanson, 1981), cats (Irle \& Markowitsch, 1982), and monkeys (Pandya, Van Hoesen, \& Mesulam, 1981; Rosene \& Van Hoesen, 1977; Schwerdtfeger, 1979). Interactions between these cortices and the hippocampus may be critical for the generation of theta activity of the hippocampus (Miller, 1991). In accordance with this, we observed high coherence values between the hippocampus and the entorhinal cortex, and the hippocampus and the cingulate cortex. This indicates that these cortical regions are actively synchronized with the hippocampus at the frequency of theta. Moreover, we also observed that these regions were in more synchronous state during the taskrelated phases than during the control phase. It is likely that these interactions at the frequency of theta activity are involved in a mnemonic process, as well as being important for the generation of hippocampal theta activity.

In addition to the comparison phase, CAl theta activity increased during the delay phase, as compared with the control phase. This may suggest that hippocampal theta activity relates to the stimulus retention process. It has also been reported that there are hippocampal cells that are differentially active during the delay phase of the DNMS task in the monkey (Colombo \& Gross, 1994) and of the modified DNMS task in the rat (Otto \& Eichenbaum, 1992b). These neuronal studies, however, showed no evidence of memory storage in the hippocampus. As for the function of the hippocampus during the delay phase, further studies are required.

With regard to the rats' performance in the present study, the probability of a go response in the match trials may be relatively high. According to our behavioral observation, the rats seemed to make a stereotyped response in the match trials. We often observed that the rats bit at the inserted lever in the match trials. This collateral behavior seemed to prevent the no-go response in the match trials from increasing. To investigate the relationship between the hippocampal theta activity and the DNMS task events, we adopted the discrete type of the DNMS task. It is likely that this type of the task was difficult for rats, because the rats' performance reached a plateau after the fourth block of training sessions (Figure 2).

Some of the conclusions and speculations expressed in the present article require further studies. First, it is necessary to confirm whether similar results are obtained in the continuous nonmatching-to-sample task, a modified version of the DNMS task (Otto \& Eichenbaum, 1992a, 1992b; Pontecorvo, 1983; Sakurai, 1987, 1990a, 1990b). Better performance could be accomplished in the continuous type. Second, it is necessary to compare the present data with theta activity during a task that has no need for mnemonic processing but that has a procedure similar to the present task (e.g., a go/no-go discrimination task). These comparisons will confirm that theta activity is involved in the mnemonic process. Third, it is necessary to investigate the laminar profile of theta activity during the task by using movable electrodes. One can record different amplitudes and different phases of theta activity in different laminar depths of the hippocampus (B. H. Bland \& Whishaw, 1976; Buzáki, 1986; Buzáki et al., 1986; Buzáki et al., 1983; Lopes da Silva, 1992). By forming these laminar profiles, it is possible to analyze in detail relationships of theta activity among the entorhinal cortex, the cingulate cortex, and the hippocampal formation, on the basis of the laminar structures. A theta generator in the cingulate cortex has been suggested but not confirmed. This means that the present cingulate theta activity might be volume-conducted from the hippocampus. The present cingulate data should be verified by using the movable electrodes. Finally, it is necessary to analyze the correlation between hippocampal theta and cortical neuronal activities by simultaneous recordings of the unit and filed potentials. Such a correlative analysis has been carried out between the hippocampal theta and the neuronal activity for the medial septal nucleus (Gaztelu \& Buño, 1982) or mammillary body (Kocsis \& Vertes, 1994). It is expected that interactions between the hippocampus and the cortex as to mnemonic functions would be manifested by an investigation of the relationship between the hippocampal theta and the neuronal activities in the entorhinal cortex, the cingulate cortex, the peri- or postrhinal cortex, the parahippocampal cortex, and the other neocortices. 


\section{REFERENCES}

Aggleton, J. P., Hunt, P. R., \& Rawlins, J. N. P. (1986). The effects of hippocampal lesions upon spatial and non-spatial tests of working memory. Behavioural Brain Research, 19, 133-146.

Alonso, A., \& García-AuStT, E. (1987a). Neuronal sources of theta rhythm in the entorhinal cortex of the rat: I. Laminar distribution of theta field potentials. Experimental Brain Research, 67, 493-501.

Alonso, A., \& García-AustT, E. (1987b). Neuronal sources of theta rhythm in the entorhinal cortex of the rat: II. Phase relations between unit discharges and theta field potentials. Experimental Brain Research, 67, 502-509.

BLAND, B. H., \& WHISHAW, I. Q. (1976). Generators and topography of hippocampal theta (RSA) in the anaesthetized and freely moving rat. Brain Research, 118, 259-280.

BLAND, S. K., \& BLAND, B. H. (1986). Medial septal modulation of hippocampal theta cell discharges. Brain Research, 375, 102-116.

BUZÁKI, G. (1986). Generation of hippocampal EEG patterns. In R. L. Isaacson \& K. H. Pribram (Eds.), The hippocampus (Vol. 3, pp. 137 167). New York: Plenum.

BuzÁki, G., CZopf, J., Kondákor, I., \& Kellényi, L. (1986). Laminar distribution of hippocampal rhythmic slow activity (RSA) in the behaving rat: Current-source density analysis, effects of urethane and atropine. Brain Research, 365, 125-137.

Buzákı, G., Leung, L. S., \& Vanderwolf, C. H. (1983). Cellular bases of hippocampal EEG in the behaving rat. Brain Research Reviews, 6, 139-171.

Colombo, M., \& Gross, C. G. (1994). Responses of inferior temporal cortex and hippocampal neurons during delayed matching-to-sample in monkeys (Macaca fascicularis). Behavioral Neuroscience, 108, 1-13.

Destrade, C., \& OTT, T. (1982). Is a retrosplenial (cingulate) pathway involved in the mediation of high frequency hippocampal thythmical slow activity (theta)? Brain Research, 252, 29-37.

Eichenbaum, H., Otto, T., \& Cohen, N. J. (1994). Two functional components of the hippocampal memory system. Behavioral \& Brain Sciences, 17, 449-518.

Feenstra, B. W. A., \& Holsheimer, J. (1979). Dipole-like neuronal sources of theta rhythm in dorsal hippocampus, dentate gyrus and cingulate cortex of the urethane-anesthetized rat. Electroencephalography \& Clinical Neurophysiology, 47, 532-538.

Ferino, F., Thierry, A. M., \& Glowinski, J. (1987). Anatomical and electrophysiological evidence for a direct projection from ammon's horn to the medial prefrontal cortex in the rat. Experimental Brain Research, 65, 421-426.

Frederickson, C. J., \& Whishaw, I. Q. (1977). Hippocampal EEG during learned and unlearned behavior in the rat. Physiology \& Behavior, 18, 597-603.

GazTeLU, J. M., \& BuÑo, JR., W. ( 1982). Septo-hippocampal relationships during EEG theta rhythm. Electroencephalography \& Clinical Neurophysiology, 54, 375-387.

Givens, B., \& OLTON, D. S. (1990). Cholinergic and GABAergic modulation of medial septal area: Effect on working memory. Behavioral Neuroscience, 104, 849-855.

GivenS, B., \& OLTON, D. S. (1994). Local modulation of basal forebrain: Effects on working memory. Journal of Neuroscience, 14, 3578-3587.

GraY, J. A. (1971). Medial septal lesions, hippocampal theta rhythm and the control of vibrissal movement in the freely moving rat. Electroencephalography \& Clinical Neurophysiology, 30, 189-197.

Green, K. F., \& RaWlins, J. N. P. (1979). Hippocampal theta in rats under urethane: Generators and phase relations. Electroencephalography \& Clinical Neurophysiology, 47, 420-429.

IrLe, E., \& Markowitsch, H. J. (1982). Connections of the hippocampal formation, mamillary bodies, anterior thalamus and cingulate cortex. Experimental Brain Research, 47, 79-94

Kocsis, B., \& VerTES, R. P. (1994). Characterization of neurons of the supramammillary nucleus and mammillary body that discharge rhythmically with the hippocampal theta rhythm in the rat. Journal of Neuroscience, 14, 7040-7052.
LEUNG, L. S., \& Borst, J. G G. (1987). Electrical activity of the cingulate cortex: I. Generating mechanisms and relations to behavior Brain Research, 407, 68-80.

LOPES DA SILVA, F. (1992). The rhythmic slow activity (theta) of the limbic cortex: An oscillation in search of a function. In E. Basar \& T. H. Bullock (Eds.), Induced rhythms in the brain (pp. 83-102). Boston: Birkhäuser.

M'Harzi, M., \& Monmaur, P. (1985). Selective lesions of the fimbria and the fornix in the rat: Differential effects on CAI and dentate theta. Experimental Neurology, 89, 361-371.

MILLER, R. (1991). Cortico-hippocampal interplay and the representation of contexts in the brain. Berlin: Springer-Verlag.

MitChELl, S. J., \& RANCK, J. B. (1980). Generation of theta rhythm in medial entorhinal cortex of freely moving rats. Brain Research, 189 , 49-66.

Monmaur, P. (1982). Hippocampal theta rhythms from CA1 and dentate generators during paradoxical sleep of the rat: Differential alterations after septal lesion. Physiology \& Behavior, 28, 467-471.

Montoya, C. P., \& Sainsbury, R. S. (1985). The effects of entorhinal cortex lesions of type 1 and type 2 theta. Physiology \& Behavior, 35 , $121-126$.

Olton, D. S., Becker, J. T., \& Handelmann, G. E. (1979). Hippocampus, space, and memory. Behavioral \& Brain Sciences, 2, 313-365.

Otto, T., \& Eichennaum, H. (1992a). Complementary roles of the orbital prefrontal cortex and the perirhinal-entorhinal cortices in an odor-guided delayed-nonmatching-to-sample task. Behavioral Neuroscience, 106, 762-775.

OTro, T., \& Eichenbaum, H. (1992b). Neuronal activity in the hippocampus during delayed non-match to sample performance in rats: Evidence for hippocampal processing in recognition memory. Hippocampus, 2, 323-334.

Pandya, D. N., Van Hoesen, G. W., \& Mesulam, M. M. (1981). Efferent connections of the cingulate gyrus in the rhesus monkey. $E x-$ perimental Brain Research, 42, 319-330.

PAXINos, G., \& WATSON, C. (1986). The rat brain in stereotaxic coordinates (2nd ed.). San Diego, CA: Academic Press.

PonteCorvo, M. J. (1983). Effects of proactive interference on rats' continuous nonmatching-to-sample performance. Animal Learning \& Behavior, 11, 356-366.

RAFFAELE, K. C., \& OLTON, D. S. (1988). Hippocampal and amygdaloid involvement in working memory for nonspatial stimuli. Behavioral Neuroscience, 102, 349-355.

Rosene, D. L., \& VAN HoEsen, G. W. (1977). Hippocampal efferents reach widespread areas of cerebral cortex and amygdala in the rhesus monkey. Science, 198, 315-317.

SaINSBURY, R. S., \& BLAND, B. H. (1981). The effects of selective septal lesions on theta production in CAl and the dentate gyrus of the hippocampus. Physiology \& Behavior, 26, 1097-1101.

SAKURAI, Y. (1987). Rats' auditory working memory tested by continuous non-matching-to-sample performance. Psychobiology, 15, 277 . 281 .

SaKuRaI, Y. (1990a). Cells in the rat auditory system have sensorydelay correlates during the performance of an auditory working memory task. Behavioral Neuroscience, 104, 856-868.

SAKURAI, Y. (1990b). Hippocampal cells have behavioral correlates during the performance of an auditory working memory task in the rat. Behavioral Neuroscience, 104, 253-263.

SCHWERDTFEgER, W. K. (1979). Direct efferent and afferent connections of the hippocampus with the neocortex in the marmoset monkey. American Journal of Physiology, 156, 77-82.

Squire, L. R. (1987). Memory and brain. Oxford: Oxford University Press.

SQUiRE, L. R. (1992). Memory and the hippocampus: A synthesis from findings with rats, monkeys, and humans. Psychological Review, 99, $165-231$.

SutherLand, R. J., \& Rudy, J. W. (1989). Configural association theory: The role of the hippocampal formation in learning, memory, and amnesia. Psychobiology, 17, 129-144. 
SWANSON, L. W. (1981). A direct projection from ammon's horn to the prefrontal cortex in the rat. Brain Research, 217, 150-154.

Troncoso, E., Rodríguez, M., \& Feria, M. (1995). Light-induced arousal affects simultaneously EEG and heart rate variability in the rat. Neuroscience Letters, 188, 167-170.

VANDERWOLF, C. H. (1969). Hippocampal electrical activity and voluntary movement in the rat. Electroencephalography \& Clinical Neurophysiology, 26, 407-418.

VanderwolF, C. H., Leung, L. S., \& CoOley, R. K. (1985). Pathways through cingulate, neo- and entorhinal cortices mediate atropine- resistant hippocampal rhythmic slow activity. Brain Research, 347, 58-73.

Winson, J. (1978). Loss of hippocampal theta rhythm results in spatial memory deficit in the rat. Science, 201, 150-163.

YouNG, G. A. (1976). Electrical activity of the dorsal hippocampus in rats operantly trained to lever press and to lick. Journal of Comparative \& Physiological Psychology, 90, 78-90.

(Manuscript received December 10, 1997; revision accepted for publication November 17, 1998.) 